\title{
Improved Randomized Approximation Algorithms for Lot-Sizing Problems
}

\author{
Chung-Piaw Teo ${ }^{1}$ Dimitris Bertsimas ${ }^{1}$
}

Sloan School of Management and Operations Research Center, MIT

\section{Introduction}

We consider in this paper multi-product, lot-sizing problems that arise in manufacturing and inventory systems. We describe the problem in a manufactruring setting. There is a set $N$ of products. For each product $j \in N$ there is a set $\pi_{j}$ (called predecessors of product $j$ ) of products consumed in producing product $j$. We define the product network $G$ to be a directed network with node set $N$ and arc set $A=\left\{(i, j): i \in \pi_{j}\right\}$. In other words, the network $G$ corresponds to the flow of materials in the system and contains no circuit.

External demand $d_{i}$ for product $i$ is assumed to be constant in time. Clearly in order to satisfy the demand orders should be placed for the products dynamically in time. If an order is placed for product $i$, an ordering cost $K_{i}$ is incurred. Moreover, an incremental echelon holding $\operatorname{cost} h_{i}$ is incurred per unit time the item spends in inventory. The production rate is assumed to be infinite. The objective is to schedule orders for each of the products over an infinite horizon so as to minimize long-run average cost.

As the optimal dynamic policy can be very complicated, the research community (see for instance Roundy [18, 19], Jackson, Maxwell and Muckstadt [10], Muckstadt and Roundy [14]) has focused on stationary and nested policies defined as follows: Orders are placed periodically in time at equal intervals, for each of the products in the system (stationary policies). If product $j$ precedes product $i$, then an order is placed for product $j$ only when an order is placed for product $i$ at the same time (nested policies). Therefore, under a stationary and nested policy the objective is to decide the period $T_{i}$ that an order is placed. The reason stationary and nested policies are attractive is that they are easy to implement. Muckstadt and Roundy [14] discuss in detail the rationale of using order intervals $T_{i}$ as variables.

The problem of designing an optimal stationary and nested policy can then be formulated (see [18]) as the following nonlinear integer programming problem.

$$
\begin{gathered}
\left(P_{N S}\right)=\min G(T) \equiv \sum_{i \in N}\left(\frac{K_{i}}{T_{i}}+H_{i} T_{i}\right) \\
\frac{T_{i}}{T_{j}} \in\{1,2,3 \ldots\} \text { if }(i, j) \in A,
\end{gathered}
$$




$$
T_{i}=k_{i} T_{L} \text { for each } i, k_{i} \in \mathcal{Z}_{+}
$$

Period $T_{L}$ is called the base period and it can be constant or allowed to vary, depending on the model. The coefficient $H_{i}$ is given by $H_{i}=\left(h_{i}-\sum_{j \in \pi_{i}} h_{j}\right) D_{i}$ and $D_{i}$ represents the aggregate demand, which is calculated recursively starting from products with $s_{i}=\emptyset$ by $D_{i}=d_{i}+\sum_{k \in s_{i}} D_{k}\left(s_{i}\right.$ is the set of successors of product $i$ ).

We consider the following convex relaxation of the problem:

$$
\begin{aligned}
\left(P_{R}\right) Z_{R}= & \min \sum_{j \in N}\left(\frac{K_{i}}{T_{i}}+H_{i} T_{i}\right) \\
T_{i} & \geq T_{j} \text { if }(i, j) \in A, \\
T_{i} & \geq T_{L} \text { for each } i .
\end{aligned}
$$

Notice that the constraints $T_{i} \geq T_{j}$ model the condition that policies are nested.

As the objective function is convex, the relaxation $\left(P_{R}\right)$ can be solved in polynomial time using interior point algorithms or the algorithm by Hochbaum and Shanktikumar [9]. For systems with special structure the runnning time can be improved substantially. For instance, if $G$ is a tree, Jackson and Roundy [11] show that the relaxed problem can be solved in $O(n \log n)$ time, where $n=|N|$, When $G$ corresponds to a star graph, Queyranne [15], and also Lu Lu and Posner [12] showed that the relaxed problem can be solved in $O(n)$ time, using a linear time median finding algorithm.

Regarding approximation algorithms, Roundy $([18,19])$, and Maxwell and Muckstadt [13] showed how to round an optimal solution of the relaxed problem $\left(P_{R}\right)$ to a feasible solution for $\left(P_{N S}\right)$. The policies constructed are called powerof-two policies, where each $T_{i}$ is of the form $2^{p_{i}} T_{L}$, where $p_{i}$ is integer. Let $Z_{H}$ be the value of the heuristic used. They obtained the following bounds:

1. If $T_{L}$ is not fixed, but subject to optimization, then

$$
\frac{Z_{H}}{Z_{R}} \leq \frac{1}{\sqrt{2} \log 2} \approx 1.02
$$

2. If $T_{L}$ is fixed, then

$$
\frac{Z_{H}}{Z_{R}} \leq\left(\sqrt{2}+\frac{1}{\sqrt{2}}\right) \approx 1.06
$$

The technique used is deterministic rounding and convex duality. The technique utilizes the properties of the optimal relaxed solution. In both cases the bounds are tight. These results are often referred in the literature as $98 \%$ and $94 \%$ effective lot-sizing policies respectively.

These results have been extensively studied and extended to other versions of lot-sizing problems: finite production rates (Atkins, Queyranne and Sun [1]), 
individual capacity bounds of the form $2^{l_{i}} T_{L} \leq T_{i} \leq 2^{u_{i}} T_{L}$ and more general cost structures (Zheng [21]), and backlog (Atkins et al. [1]). All these extensions use determinisitc rounding to generate power-of-two policies with the same $94 \%$ and $98 \%$ bounds.

In this paper, we propose a new approach to these lot-sizing problems that uses randomized rounding. This design technique has been used extensively by the discrete optimization community. It was first introduced by Raghavan and Thompson [16], and was used subsequently for a variety of other combinatorial problems. See for instance Goemans and Williamson [7, 8], Bertsimas and Vohra [3], Bertsimas et al. [2]. Our contributions in this paper are as follows:

1. We propose new $94 \%$ and $98 \%$ randomized rounding algorithms for Problem $\left(P_{N S}\right)$ under both the fixed and the variable base period models. Our proof is simple and unlike the original deterministic rounding does not depend on the structure of the optimal solution. Roundy's $98 \%$ algorithm can be obtained by derandomizing our algorithm. However, derandomizing the $94 \%$ algorithm leads to a different deterministic algorithm. The randomized rounding method is interesting in its own right as it introduces dependencies in the rounding process and generates random variables with distributions with nonlinear density functions.

2. We study a generalization of the fixed based model by allowing the base period $T_{L}$ to vary over a finite set of choices $\left\{2^{k / p} T_{L}: k\right.$ integer $\}$, with $p, T_{L}$ fixed. We propose a randomized rounding algorithm that produces a power-of two policy with bound $\frac{2^{\frac{1}{p}}+1}{2 \sqrt{2} p\left(2^{\frac{1}{p}}-1\right)}$, where $p$ denote the number of points $T_{L}$ is allowed to vary. For $p=1$ and $p=\infty$, the bound reduces to 1.06 and 1.02 respectively. For the one warehouse, multi-retailer problem (OWMR), Lu Lu and Posner [12] have also obtained a similar bound for a class of integer-ratio policies.

3. For a general production distribution network under nested policies, we propose new convex relaxations and randomized rounding algorithms that use $T_{i}=2^{p_{i}} T_{L}$ or $3 \cdot 2^{p_{i}} T_{L}$. This improves the bound for the fixed base period case from 1.06 to 1.043 and for the special case of Problem (OWMR) to 1.031 .

4. Our techniques generalize to several other extensions considered in the literature (capacitated versions, submodular cost functions and multiple resource constrained problems) 


\section{Randomized rounding and lot-sizing prob- lems}

In this section, we introduce the key randomized rounding ideas used in this paper.

\subsection{A new 94\% approximation algorithm}

In this section we consider the case of fixed base period $T_{L}$. We consider the following rounding scheme:

\section{Algorithm A:}

Let $T=\left(T_{1}, \ldots, T_{n}\right)$ be a feasible solution to relaxation $\left(P_{R}\right)$, and $T_{i}=2^{p_{i}} z_{i} T_{L}$, where $1 \leq z_{i} \leq 2$. Generate a point $Y$ in the interval $[1,2]$, with probability distribution $F(y)=\frac{y^{2}-1}{1+y^{2} / 2}$. If $z_{i}<Y$, then $T_{i}^{o}=2^{p_{i}} T_{L}$, else $T_{i}^{0}=2^{p_{i}+1} T_{L}$.

The above rounding scheme always generates a feasible solution $\left(T_{1}^{o}, T_{2}^{o}, \ldots, T_{n}^{o}\right)$ to problem $\left(P_{N S}\right)$. We only need to check that the precedence constraints $T_{i} \leq T_{j}$ are preserved. If $p_{j}>p_{i}$, then $T_{j}^{o} \geq T_{i}^{o}$. If $p_{j}=p_{i}$, then since $T_{j} \geq T_{i}$, we must have $z_{j} \geq z_{i}$. Hence $z_{i} \geq y$ only if $z_{j} \geq y$.

Theorem 1. Given any feasible solution $\left(T_{1}, \ldots, T_{n}\right)$ to Problem $\left(P_{R}\right)$ with cost $G(T)$, Algorithm A returns a power-of-two policy (with fixed base $T_{L}$ ) with an expected cost of not more than $1.06 \mathrm{G}(T)$.

Proof: It is easy to see that

$$
\begin{aligned}
E\left(T_{i}^{o}\right)= & 2^{p_{i}} T_{L}\left(1-F\left(z_{i}\right)\right)+2^{p_{i}+1} T_{L} F\left(z_{i}\right)=T_{i} \frac{1+F\left(z_{i}\right)}{z_{i}} \\
& =T_{i} \frac{3 z_{i}}{z_{i}^{2}+2} \leq \frac{\sqrt{2}+1 / \sqrt{2}}{2} T_{i} \approx 1.06 T_{i} .
\end{aligned}
$$

Similarly,

$$
\begin{gathered}
E\left(\frac{1}{T_{i}^{o}}\right)=\frac{1}{2^{p_{i} T_{L}}}\left(1-F\left(z_{i}\right)\right)+\frac{1}{2^{p_{i}+1} T_{L}} F\left(z_{i}\right)=\frac{1}{T_{i}}\left(1-\frac{F\left(z_{i}\right)}{2}\right) z_{i} \\
=\frac{1}{T_{i}} \frac{3 z_{i}}{z_{i}^{2}+2} \leq \frac{\sqrt{2}+1 / \sqrt{2}}{2} \frac{1}{T_{i}} .
\end{gathered}
$$

The bound follows since the maximum value of the function $\frac{3 z_{i}}{z_{i}^{2}+2}$ is at most $3 \sqrt{2} / 4$. 
Note that the distribution function $F(y)$ is chosen so that $(1+F(y)) / y=$ $y(1-F(y) / 2)=3 y /\left(y^{2}+2\right)$. The maximum is attained at the point $y=\sqrt{2}$ with a value of $\frac{3 \sqrt{2}}{4} \approx 1.06$. Furthermore, using the optimal solution to $\left(P_{R}\right)$ as input to the rounding process, we obtain a $94 \%$ approximation algorithm to the original lot-sizing problem.

De-randomization. The above randomized algorithm can be made deterministic: Sort the $z_{i}$ 's in non-decreasing order, say $z_{1} \leq z_{2} \leq \ldots \leq z_{n}$. For all $y$ in $\left[z_{i}, z_{i+1}\right]$, the randomized algorithm returns the same solution. Hence, there are at most $n+1$ distinct solutions obtained. Thus the best solution can be obtained in $O(n \log n)$ time, which is the time needed for the sorting operation.

\subsection{The $98 \%$ approximation algorithm revisited}

The same insensitivity result can also be improved to a $98 \%$ guarantee, if one allows the base period $T_{L}$ to vary, i.e., $T_{L}$ is a variable in $\left(P_{R}\right)$. In fact, Roundy's $98 \%$ algorithm $[18,19]$ already has this feature. We recast Roundy's algorithm into the following randomized rounding algorithm:

\section{Algorithm B:}

Let $T=\left(T_{1}, \ldots, T_{n}, T_{L}\right)$ be a feasible solution to $\left(P_{R}\right)$, with $T_{L}>0$.

Let $T_{i}=2^{p_{i}} T_{L} z_{i}$, where $1 \leq z_{i} \leq 2$. Generate a point $Y$ in the interval [1,2], with probability distribution $F(y)=\frac{\log y}{\log 2}$. If $Y>z_{i}$, then $T_{i}^{o}=2^{p_{i}} \frac{Y}{\sqrt{2}}$ else $T_{i}^{o}=2^{p_{i}+1} \frac{Y}{\sqrt{2}}$. Let $T_{L}^{o}=\frac{Y}{\sqrt{2} T_{L}}$.

The rounded solution $T_{i}^{o}$ is chosen to ensure that it lies in the interval $\left[\frac{T_{i}}{\sqrt{2}}, \sqrt{2} T_{i}\right]$. Furthermore, it is clear that $\left(T_{1}^{\circ}, T_{2}^{o}, \ldots, T_{n}^{o}, T_{L}^{o}\right)$ is a feasible solution to $\left(P_{N S}\right)$.

Theorem 2. Given any feasible solution $\left(T_{1}, \ldots, T_{n}, T_{L}\right)$ to Problem $\left(P_{R}\right)$ with cost $G(T)$, Algorithm $B$ returns a power-of-two policy $\left(T_{1}^{o}, T_{2}^{o}, \ldots, T_{n}^{o}, T_{L}^{o}\right)$ with expected cost at most $\frac{G(T)}{\sqrt{2} \log (2)} \approx 1.02 G(T)$.

Proof: Without loss of generality, we may assume $T_{L}=1$. Then

$$
\begin{gathered}
E\left(T_{i}^{o}\right)=\frac{\int_{1}^{z_{i}} 2^{p_{i}+1} d y+\int_{z_{i}}^{2} 2^{p_{i}} d y}{\sqrt{2} \log 2} \\
=\frac{2^{p_{i}}\left[2\left(z_{i}-1\right)+\left(2-z_{i}\right)\right]}{\sqrt{2} \log 2}=\frac{T_{i}}{\log 2 \sqrt{2}} .
\end{gathered}
$$

Similarly,

$$
E\left(1 / T_{i}^{o}\right)=\frac{\sqrt{2} \int_{1}^{z_{i}} 2^{-p_{i}-1}\left(1 / y^{2}\right) d y+\sqrt{2} \int_{z_{i}}^{2} 2^{-p_{i}}\left(1 / y^{2}\right) d y}{\log 2}
$$




$$
=\frac{\sqrt{2} 2^{-p_{i}}\left(1 / 2-\frac{1}{2 z_{i}}-1 / 2+\frac{1}{z_{i}}\right)}{\log 2}=\frac{1}{T_{i} \log 2 \sqrt{2}},
$$

and the theorem follows.

De-randomization. Suppose $z_{1} \leq z_{2} \leq \ldots \leq z_{n}$. For $y$ in $\left[z_{i}, z_{i+1}\right)$, suppose the algorithm returns a policy with cost $A / y+B y$, then for all other $y^{\prime}$ in the same interval, the algorithm returns a policy with cost $A / y^{\prime}+B y^{\prime}$. By choosing a $y^{\prime}$ in the interval that maximizes this term, and doing the same for each interval partitioned by the $z_{i}$ 's, we obtained an $O(n \log n)$ deterministic algorithm, which is exactly Roundy's rounding procedure.

The argument used above can easily be adapted to analyze more general costs in the objective function. For instance, we have the following:

Theorem 3. Under Algorithm B,

$$
\begin{gathered}
E\left\{\left(T_{i}^{o}\right)^{2}\right\} / T_{i}^{2}=T_{i}^{2} E\left\{\left(\frac{1}{T_{i}^{o}}\right)^{2}\right\}=\frac{3}{4 \log (2)} \approx 1.082 ; \\
\frac{1}{\sqrt{2} \log (2)} \leq E\left(\frac{1}{T_{i}^{o} T_{j}^{o}}\right) T_{i} T_{j} \leq \frac{3}{4 \log (2)} ; \\
\frac{1}{\sqrt{2} \log (2)} \leq E\left(T_{i}^{o} T_{j}^{o}\right) \frac{1}{T_{i} T_{j}} \leq \frac{3}{4 \log (2)} ; \\
E\left(\frac{T_{i}^{o}}{T_{j}^{o}}\right) \leq 1.06 \frac{T_{i}}{T_{j}} .
\end{gathered}
$$

The above inequalities imply new bounds $(91.8 \%)$ if there are $T_{i}^{2}, \frac{1}{T_{i}^{2}}, T_{i} T_{j}$ or $\frac{T_{i}}{T_{j}}$ terms in the objective function.

\subsection{Unification of the $94 \%$ and $98 \%$ bounds}

The $94 \%$ and $98 \%$ performance bounds assume that the base period is fixed and optimally selected respectively. The $94 \%$ bound is attained by a power-oftwo policy, where every order interval is a fixed multiple of a preselected base period. The $98 \%$ approximation algorithm, however, cannot ensure that the base planning period belongs to a preselected set. In this section, we propose a technique to bridge the gap between the performance of these two algorithms, by giving progressively more flexibility to the choice of base periods. We assume that the allowed base periods are in the set $\mathcal{S}=\left\{2^{\frac{j}{p}}: j\right.$ integer $\}$.

Consider the following randomized rounding algorithm. 


\section{Algorithm C:}

Let $T_{i}=2^{p_{i}} z_{i}$, where $1 \leq z_{i}<2$. Let $Y$ be a random number generated in the interval $\left[2^{-\frac{1}{2 p}}, 2^{\frac{1}{2 p}}\right)$, with distribution function $F(y)=\frac{2^{1 / p} y^{2}-1}{\left(2^{1 / p}-1\right)\left(1+y^{2}\right)}$. Construct a power-of-two policy as follows:

Select the base period $T_{L}=2^{j / p}$ with probability $\frac{1}{p}$.

$$
T_{i}^{o}= \begin{cases}2^{p_{i}+1} 2^{\frac{j}{p}} & \text { if } z_{i}>\sqrt{2} 2^{\frac{j}{p}} Y \\ 2^{p_{i}-1} 2^{\frac{i}{p}} & \text { if } z_{i}<\frac{2^{\frac{i}{p}} Y}{\sqrt{2}} \\ 2^{p_{i}} 2^{\frac{i}{p}} & \text { otherwise. }\end{cases}
$$

Theorem 4. Given any feasible lot-sizing policy $\left(T_{1}, \ldots, T_{n}\right)$ in $\left(P_{R}\right)$ with cost $G(T)$, Algorithm $C$ returns a power-of-two policy $T^{o}$ with expected cost at most

$$
\left(\frac{2^{\frac{1}{p}}+1}{2 \sqrt{2} p\left(2^{\frac{1}{p}}-1\right)}\right) G(T) .
$$

Note that for $p=1$ and $p=\infty$, we obtain the $94 \%$ and $98 \%$ bounds respectively. For $p=2$, the bound already improves to $97 \%$. This observation implies that for the fixed base period model, the $94 \%$ bound might be improved considerably by considering only two distinct base periods, both integral multiples of $T_{L}$. In the next section, we use this observation to derive an improved approximation algorithm.

\section{An improved approximation algorithm for the fixed base period model}

In this section, we propose an improved approximation algorithm for the general problem $\left(P_{N S}\right)$ under the fixed base period model. The improvement over the $94 \%$ bound comes from having a tighter representation of the objective function over the discrete points $\left\{T_{L}, 2 T_{L}, 3 T_{L}\right\}$ in the interval $\left[T_{L}, 3 T_{L}\right]$. We consider an improved relaxation of the original problem:

$$
\begin{gathered}
\left(P_{R}^{\prime}\right) \min \sum_{i=1}^{n} f_{i}^{\prime}\left(T_{i}\right) \\
\text { subject to } T_{i} \geq T_{j} \text { if }(i, j) \in A, T_{i} \geq 0,
\end{gathered}
$$

where $f^{\prime}(\cdot)$, which is depicted in Figure 1 , represents the piecewise linearization of $f_{i}\left(T_{i}\right)=K_{i} / T_{i}+H_{i} T_{i}$ over the points $\left\{T_{L}, 2 T_{L}, 3 T_{L}\right\}$, i.e.,

$$
f_{i}^{\prime}\left(T_{i}\right)= \begin{cases}\left(T_{i}-T_{L}\right) f_{i}\left(2 T_{L}\right)+\left(2 T_{L}-T_{i}\right) f_{i}\left(T_{L}\right) & \text { if } T_{L} \leq T_{i} \leq 2 T_{L} \\ \left(T_{i}-2 T_{L}\right) f_{i}\left(3 T_{L}\right)+\left(3 T_{L}-T_{i}\right) f_{i}\left(2 T_{L}\right) & \text { if } 2 T_{L} \leq T_{i} \leq 3 T_{L}\end{cases}
$$




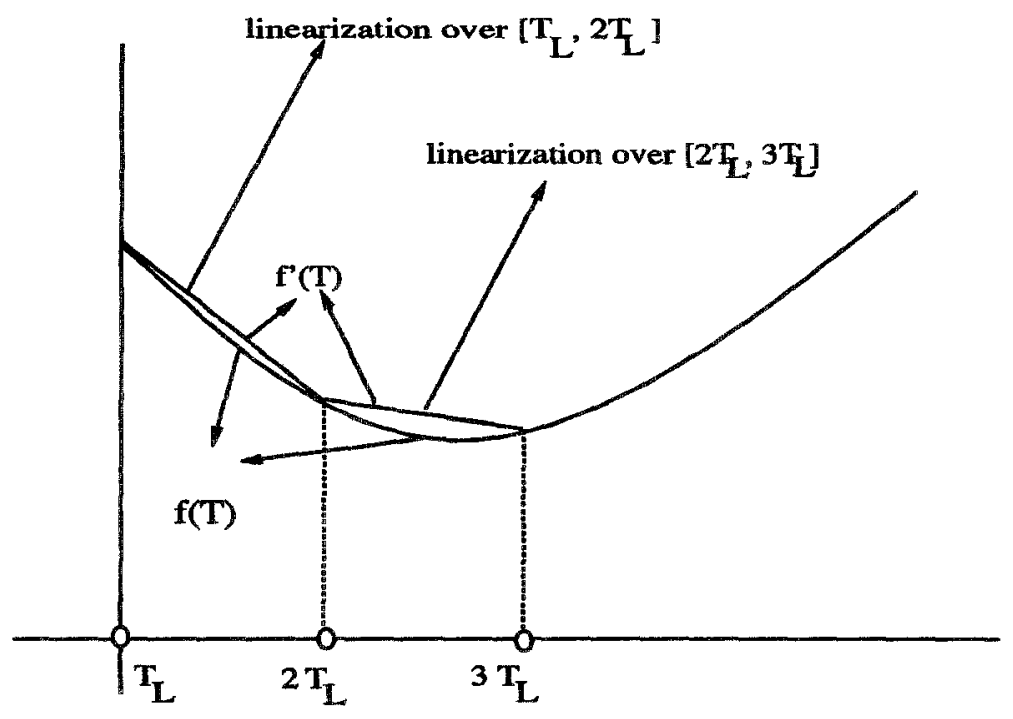

Fig. 1. Piecewise linearization of the objective function over the points $T_{L}, 2 T_{L}, 3 T_{L}$.

We introduce the following notation. Let $p, q$ be nonnegative numbers, such that $p+q=1$. Let

$$
a(p)=2 \sqrt{\frac{p+3 q / 2}{p+2 q / 3}}, b(p)=2 \sqrt{\frac{2 p+3 q / 2}{p / 2+2 q / 3}},
$$

and

$$
\begin{aligned}
& F(p, z)=\frac{\frac{1}{4}\left(p+\frac{2}{3} q\right) z^{2}-\frac{3}{2} q-p}{p\left(1+\frac{1}{8} z^{2}\right)}, \\
& F^{\prime}(p, z)=\frac{\frac{1}{9}\left(q+\frac{3}{4} p\right) z^{2}-\frac{4}{3} p-q}{q\left(1+\frac{1}{18} z^{2}\right)} .
\end{aligned}
$$

Note that $F(p, a(p))=F^{\prime}(p, b(p))=0$, and $F(p, b(p))=F^{\prime}(p, 2 a(p))=1$. Furthermore, $F$ and $F^{\prime}$ are nondecreasing in $z$ and are valid distribution functions. Suppose further that $T^{*}$ is an optimal solution to $\left(P_{R}^{\prime}\right)$. Note that for $T_{i}^{*} \leq 3 T_{L}$, we may assume that $T_{i}^{*} \in\left\{T_{L}, 2 T_{L}, 3 T_{L}\right\}$. This follows from the following lemma:

Lemma 5. There exists an optimal solution $T^{*}$ with the property that $T_{i}^{*} \in$ $\left\{T_{L}, 2 T_{L}, 3 T_{L}\right\}$ if $T_{i}^{*} \leq 3 T_{L}$. 
Consider the following rounding algorithm:

Algorithm D: Let $p=0.7, q=0.3$. Let $a=a(0.7)$ and $b=b(0.3)$. Note that $a<b<2 a$. Select Policy 1 below with probability $p$, and Policy 2 with probability $q$.

Policy 1: Let $T_{i}^{*}=2^{p_{i}} T_{L} z_{i}$, where $z_{i}$ is in the interval [1,2). Let $Y$ be a random number generated in the interval $[a(p), b(p)]$ with distribution function $F(p, y)$. Let

$$
T_{i}^{1}= \begin{cases}2^{p_{i}} T_{L} & \text { if } 2 z_{i}<Y, \\ 2^{p_{i}+1} T_{L} & \text { if } 2 z_{i} \geq Y .\end{cases}
$$

Policy 2: Let $T_{i}^{*}=3 \cdot 2^{p_{i}} T_{L} z_{i}^{\prime}$, where $z_{i}^{\prime}$ is in the interval $[1,2)$. Let $Y^{\prime}$ be a random number generated in the interval $[b(p), 2 a(p)]$ with distribution function $F^{\prime}(p, y)$. For $T_{i} \geq 3 T_{L}$, let

$$
T_{i}^{2}= \begin{cases}3 \cdot 2^{p_{i}} T_{L} & \text { if } 3 z_{i}^{\prime}<Y^{\prime}, \\ 3 \cdot 2^{p_{i}+1} T_{L} & \text { if } 3 z_{i}^{\prime} \geq Y^{\prime}\end{cases}
$$

For all items $i$ with $T_{i}=2 T_{L}$, we round them (simultaneously) to $3 T_{L}$ with probability $\frac{9}{14}$ and to $T_{L}$ with probability $\frac{5}{14}$. Note that in this way, for $T_{i}=2 T_{L}$,

$$
\frac{E\left(T_{i}^{2}\right)}{2 T_{L}}=\frac{8}{7}=2 T_{L} E\left(\frac{1}{T_{i}^{2}}\right) .
$$

Finally, if $T_{i}^{*}=T_{L}, T_{i}^{2}=T_{L}$.

Let $T$ denote the vector of ordering intervals under the selected policy.

Theorem 6. The expected cost of the policy $T$ produced by algorithm $D$ is at most 1.043 times the value of the continuous relaxation $\left(P_{R}^{\prime}\right)$.

Proof: Without loss of generality, we assume $T_{L}=1$. If $T_{i}^{*}=2$, then

$$
\frac{E\left(T_{i}\right)}{2 T_{L}}=2 T_{L} E\left(\frac{1}{T_{i}}\right)=p+\frac{8}{7} q=1.0428 \ldots
$$

Thus we only need to consider the case when $T_{i}^{*}$ greater than 3 . Suppose $T_{i}^{*}$ lies in (1) $\left[2^{k_{i}} a, 2^{k_{i}} b\right]$ or (2) $\left(2^{k_{i}} b, 2^{k_{i}+1} a\right]$. In case (1), Policy 2 always rounds $T_{i}$ to $3 \cdot 2^{k_{i}}$, whereas in case (2), Policy 1 always rounds $T_{i}$ to $2^{k_{i}+2}$.

Case (1): $T_{i}^{*}$ lies in $\left[2^{p_{i}} a, 2^{p_{i}} b\right]$, i.e., $T_{i}^{*}=2^{p_{i}} w_{i}$, where $w_{i} \in[a, b]$. Then

$$
E\left(T_{i}\right)=p E\left(T_{i}^{1}\right)+q E\left(T_{i}^{2}\right)=T_{i}^{*}\left(\frac{3 q}{w_{i}}+p \frac{2\left(1+F\left(p, w_{i}\right)\right)}{w_{i}}\right),
$$


and

$$
E\left(\frac{1}{T_{i}}\right)=p E\left(\frac{1}{T_{i}^{1}}\right)+q E\left(\frac{1}{T_{i}^{2}}\right)=\frac{1}{T_{i}^{*}}\left(q \frac{w_{i}}{3}+p\left(1-\frac{F\left(p, w_{i}\right)}{2}\right) w_{i} / 2\right) .
$$

We have chosen $F(p, \cdot)$ such that

$$
q \frac{3}{w_{i}}+p \frac{2\left(1+F\left(p, w_{i}\right)\right)}{w_{i}}=q \frac{w_{i}}{3}+p\left(1-\frac{F\left(p, w_{i}\right)}{2}\right) w_{i} / 2 .
$$

With this choice of $F(p, \cdot)$, and $p=0.7, q=0.3$, we can optimize the bound over the range of $w_{i}$ to obtain

$$
\frac{E\left(T_{i}\right)}{T_{i}^{*}}=T_{i}^{*} E\left(\frac{1}{T_{i}}\right) \leq 1.043
$$

Case (2) : $T_{i}^{*}$ lies in $\left(2^{p_{i}} b, 2^{p_{i}+1} a\right]$, i.e. $T_{i}^{*}=2^{p_{i}} w_{i}$ where $w_{i} \in(b, 2 a]$. Then

$$
E\left(p T_{i}^{1}+q T_{i}^{2}\right)=T_{i}^{*}\left(p \frac{4}{3 w_{i}}+q \frac{3\left(1+F^{\prime}\left(p, w_{i}\right)\right)}{w_{i}}\right),
$$

and

$$
E\left(p \frac{1}{T_{i}^{1}}+q \frac{1}{T_{i}^{2}}\right)=\frac{1}{T_{i}^{*}}\left(p \frac{3 w_{i}}{4}+q\left(1-\frac{F^{\prime}\left(p, w_{i}\right)}{2}\right) w_{i} / 3\right) .
$$

We have chosen $F^{\prime}(p, \cdot)$ such that

$$
p \frac{4}{3 w_{i}}+q \frac{3\left(1+F^{\prime}\left(p, w_{i}\right)\right)}{w_{i}}=p \frac{3 w_{i}}{4}+q\left(1-\frac{F^{\prime}\left(p, w_{i}\right)}{2}\right) w_{i} / 3 .
$$

With this choice of $F^{\prime}(p, \cdot)$, again we have

$$
\frac{E\left(p T_{i}^{1}+q T_{i}^{2}\right)}{T_{i}^{*}}=T_{i}^{*} E\left(p \frac{1}{T_{i}^{1}}+q \frac{1}{T_{i}^{2}}\right) \leq 1.043 .
$$

Hence the result follows.

We next show that if $T_{i}^{*} \geq \sqrt{6} T_{L}$ for all $i$ we can improve the approximation guarantee. This result will be useful in the next section. We consider the following modified rounding algorithm:

Algorithm E: Let $p=0.5, q=0.5$. Select Policy 1 with probability $p$ and Policy 2 otherwise:

Policy 1: The same as in Algorithm D.

Policy 2: For $T_{i}^{*} \geq 3 T_{L}$, the same as in Algorithm D. For $T_{i}^{*}$ in $\left[\sqrt{6} T_{L}, 3 T_{L}\right]$, we round $T_{i}$ to $3 T_{L}$.

The following result follows from a similar analysis to Theorem 6 .

Theorem 7. If $T_{i}^{*} \geq \sqrt{6} T_{L}$ for all $i$, then the expected cost of the policy $T$ obtained from Algorithm $E$ is at most 1.031 times the optimal value of the continuous relaxation $\left(P_{R}\right)$. 


\section{An improved approximation algorithm for the (OWMR) problem}

In this section we improve the guarantee of 1.043 to 1.031 for the problem of a single warehouse supplying and distributing items to a group of $\boldsymbol{n}$ retailers. For distribution systems Roundy [18] has showed that the optimal nested policy can be arbitrarily bad compared to the optimal stationary policy. Under the assumption that the retailers place their order only when their inventory level is zero, he showed that there is an optimal stationary policy which satisfies the integer ratio property, i.e., the ratio of the ordering interval $T_{i}$ for retailer $i$ and the ordering interval $T_{0}$ of the warehouse is either an integer or 1 over an integer. He has also constructed similar $94 \%$ and $98 \%$ approximation algorithms for problem $(O W M R)$, with fixed and variable base period respectively.

The problem can be modelled as follows (see [18]):

$$
\begin{gathered}
\left(P_{\text {OWMR }}\right) \min C(T)=\sum_{i=0}^{n}\left(K_{i} / T_{i}\right)+\sum_{i=1}^{n}\left(g_{i} \max \left(T_{0}, T_{i}\right)+H_{i} T_{i}\right) \\
\text { subject to } \frac{T_{i}}{T_{0}} \in\left\{k_{i}, \frac{1}{k_{i}}: k_{i} \text { integer }\right\}, \\
\frac{T_{i}}{T_{L}} \text { integer for all } i=0, \ldots, n,
\end{gathered}
$$

where $g_{i}=\frac{1}{2} h_{0} d_{i}$, and $H_{i}=\frac{1}{2}\left(h_{i}-h_{0}\right) d_{i}$. We consider the following relaxation:

$$
\left(P_{\text {ROWMR }}\right) \quad \min \sum_{i=0}^{n}\left(K_{i} / T_{i}\right)+\sum_{i=1}^{n}\left(g_{i} \max \left(T_{0}, T_{i}\right)+H_{i} T_{i}\right)
$$

subject to $T_{i} \geq T_{L}$ for all $i=0, \ldots, n$.

The constraint $T_{i} \geq T_{L}$ is a relaxation of the condition that each $T_{i}$ is an integral multiple of $T_{L}$. Let $T_{i}^{*}, i=0,1, \ldots, n$ be a solution of the relaxation $\left(P_{R O W M R}\right)$.

In this section, we improve on the approximation bound for the fixed base period model, by using six stronger relaxations. These relaxations correspond to the requirement that either $T_{0}^{*} \geq 6 T_{L}$ or $T_{0}^{*}=k T_{L}$ for $k$ in $\{1,2,3,4,5\}$.

We first consider the relaxation

$$
\text { (P) } Z_{6}=\min \left\{\sum_{i=1}^{n}\left(f_{i}^{\prime}\left(T_{i}\right)+g_{i} \max \left(T_{0}, T_{i}\right)\right)+K_{0} / T_{0}: T_{0} \geq 6 T_{L}, T_{i} \geq T_{L}\right\}
$$

where $f_{i}^{\prime}\left(T_{i}\right)=f_{i}\left(T_{i}\right)=K_{i} / T_{i}+H_{i} T_{i}$ if $T_{i} \geq 3 T_{L}$, and $f_{i}^{\prime}\left(T_{i}\right)$ is the piecewise linearization of $f_{i}\left(T_{i}\right)$ over the points $\left\{T_{L}, 2 T_{L}, 3 T_{L}\right\}$. Note that this relaxation provides a lower bound to the optimal value of $\left(P_{O W M R}\right) . Z_{6}$ can be computed in $O(n)$ time by using a linear time median finding algorithm, as suggested in 
Queyranne [15] or Lu Lu and Posner [12]. Let $T_{i}^{*}$ be the optimal solution of relaxation $\left(P_{6}\right)$. Following Lemma 5 we may assume that $T_{i}^{*} \in\left\{T_{L}, 2 T_{L}, 3 T_{L}\right\}$ if $T_{i}^{*} \leq 3 T_{L}$. We apply Algorithm $E$ that leaves those $T_{i}^{*}$ with values $T_{L}$ or $2 T_{L}$ unchanged.

Lemma 8. Algorithm $E$ applied to an optimal solution to relaxation $\left(P_{6}\right)$ produces an integer ratio policy with cost within 1.031 of $Z_{6}$.

Proof: Policies 1 and 2 of Algorithm $\mathrm{E}$ round those $T_{i}^{*}$ with values greater than or equal to $3 T_{L}$ to a power-of-two policy of the type $2^{p_{i}} T_{L}$ or $2^{p_{i}}\left(3 T_{L}\right)$. Those $T_{i}^{*}$ with values $T_{L}$ or $2 T_{L}$ are left unchanged. The expected gap between $T_{i}^{*}$ and the rounded value $T_{i}$ again satisfies

$$
\frac{E\left(T_{i}\right)}{T_{i}^{*}} \leq 1.031, \frac{E\left(1 / T_{i}\right)}{1 / T_{i}^{*}} \leq 1.031
$$

Note that in addition, because of the dependence in the rounding process,

$$
\left.E\left[\max \left(T_{i}, T_{0}\right)\right]=\max \left(E\left[T_{i}\right], E\left[T_{0}\right]\right)\right] \leq 1.031 \max \left(T_{i}^{*}, T_{0}^{*}\right) .
$$

Note that since $T_{0}^{*} \geq 6 T_{L}, T_{0}^{*}$ is rounded to a multiple of $4 T_{L}$ (under Policy 1) or multiples of $6 T_{L}$ (under Policy 2). Therefore, the policy constructed need not satisfy the condition $T_{0} \geq 6 T_{L}$, since Policy 1 might round $T_{0}^{*}$ down to $4 T_{L}$. However, the policy obtained is an integer ratio policy.

We next consider the case that $T_{0}^{*}=k T_{L}, k \in\{1,2,3,4,5\}$. Let $f_{i}^{k}\left(T_{i}\right)$ denote a partial piecewise linearization of $f_{i}\left(T_{i}\right)$ in the interval $\left[T_{L}, 3 k T_{L}\right]$, over the points $T_{L}, k T_{L}, 2 k T_{L}, 3 k T_{L}$. Particularly for $k=4$, in addition to $T_{L}, 4 T_{L}, 8 T_{L}, 12 T_{L}$ we include the point $2 T_{L}$ in the linearization. For $k \in\{1,2,3,4,5\}$ we consider the following five relaxations, in which we fix the value of $T_{0}$ to be $k T_{L}$ and consider the linearization $f_{i}^{k}\left(T_{i}\right)$ instead of $f_{i}\left(T_{i}\right)$ :

$\left(P_{k}\right) Z_{k}=\min \left\{C^{k}(T)=K_{0} /\left(k T_{L}\right)+\sum_{i=1}^{n}\left(g_{i} \max \left(k T_{L}, T_{i}\right)+f_{i}^{k}\left(T_{i}\right)\right): T_{i} \geq T_{L}\right\}$.

Note that each relaxation can be solved in $O(n)$. Moreover,

Lemma 9. There exists an optimal solution $T^{k}$ to $Z_{k}$ with the property that if $T_{i}^{k} \leq 3 k T_{L}$, then

$$
\begin{gathered}
T_{i}^{k} \in\left\{T_{L}, k T_{L}, 2 k T_{L}, 3 k T_{L}\right\} \text { for } k=1,2,3,5 \\
T_{i}^{k} \in\left\{T_{L}, 2 T_{L}, 4 T_{L}, 8 T_{L}, 12 T_{L}\right\} \text { for } k=4 .
\end{gathered}
$$

We next show that Algorithm $\mathrm{E}$ applied to the optimal solution of relaxation $\left(P_{k}\right)$ produces an integer ratio policy within 1.031 of $Z_{k}$. 
Lemma 10. For $k=1, \ldots, 5$ Algorithm $E$ applied to an optimal solution of relaxation $\left(P_{k}\right)$ that satisfies Lemma 9 produces an integer ratio policy with cost within 1.031 of $Z_{k}$.

Combining Lemmas 8 and 10 we obtain

Theorem 11. For the one-warehouse-multi-retailer problem with fixed base period, there is an $O(n)$ time $96.9 \%$ approximation algorithm.

\section{Extensions}

Since our prior analysis did not utilize any structure of the optimal solution, our proof techniques cover several extensions of the basic models almost effortlessly. Our techniques produce randomized rounding algorithms for the following problems considered in the literature:

1. Capacitated lot-sizing problems, in which we add constraints $2^{I_{i}} T_{L} \leq T_{i} \leq$ $2^{u_{i}} T_{L}$ for each $i$. Since the Algorithms A and B preserve these properties, Theorems 1 and 2 apply also for this capacitated version of the problem, giving rise to $94 \%$ and $98 \%$ power-of-two policies respectively. The same result was also derived in Federgruen and Zheng [6] by extending Roundy's approach to the capacitated version.

2. Submodular ordering costs introduced in Federgruen et al. [5] and Zheng [21]:

$$
\begin{gathered}
\left(P_{S U B}\right) Z=\min _{T} \max _{k} \sum_{j \in N}\left(\frac{k_{i}}{T_{i}}+H_{i} T_{i}\right) \\
T_{i} \leq T_{j} \text { if }(i, j) \in A, \\
T_{i} \geq T_{L} \text { for each } i . \\
k \in \mathcal{P},
\end{gathered}
$$

where

$$
\mathcal{P}=\left\{k: \sum_{j \in S} k_{j} \leq K(S), \sum_{j \in N} k_{j}=K(N), k_{j} \geq 0\right\},
$$

and $K(S)$ submodular. Algorithms A and B can be used to round the fractional optimal solution in $\left(P_{S U B}\right)$ to $94 \%$ and $98 \%$ optimal power-oftwo solutions. Furthermore, if $T_{i}^{*} \geq \sqrt{6} T_{L}$ for all $i$, then the fixed base period bound can be improved further to $96.9 \%$, using Theorem 7 .

3. Resource constrained lot-sizing problems considered in Roundy [20], in which we add to $\left(P_{N S}\right)$ constraints of the type

$$
\sum_{j} a_{i j} / T_{j} \leq A_{i}, \quad i=1, \ldots, m .
$$


He showed that there is a power-of-two policy for the variable base period case with cost at most 1.44 times the optimal solution. We can generalize this result to the lot-sizing problems with submodular joint cost function. Consider the following algorithm:

\section{Algorithm F:}

Let $\left(k^{*}, T^{*}\right)$ be an optimal solution to $\left(P_{S U B}\right)$ with the resource constraints added. Use $T_{j}=\sqrt{2} T_{j}^{*}$ in Algorithm B to obtain a power-of-two policy $T^{\theta}$.

First note that $T_{j}^{o}$ lies in the interval $\left[T_{j} / \sqrt{2}, T_{j} \sqrt{2}\right]$ and hence $T_{j}^{o} \geq T_{j}^{*}$. Therefore, $T_{j}^{o}$ satisfies the resource constraints.

Theorem 12. Let $T^{*}$ be an optimal solution to the resource constrained version of $\left(P_{S U B}\right)$. Using Algorithm $F$ on $T^{*}$, we obtain a power-of-two policy with cost at most 1.44 times of the optimal.

Proof: Since scaling by $\sqrt{2}$ does not affect the ordering of $T_{j}^{*}$, the solution $k^{*}$ is also a maximum solution to $G\left(T^{\circ}\right)$. Therefore, the result follows directly from the following observation:

$$
E\left(T_{j}^{o}\right) \leq \frac{1}{\sqrt{2} \log (2)} T_{j}=\frac{1}{\sqrt{2} \log (2)} \sqrt{2} T_{j}^{*} \approx 1.44 T_{j}^{*}
$$

and

$$
E\left(\frac{1}{T_{j}^{o}}\right) \leq \frac{1}{\sqrt{2} \log (2) T_{j}} \leq \frac{1}{T_{j}^{*}}
$$

\section{References}

1. D. Atkins, M. Queyranne and D. Sun. Lot sizing policies for finite production rate assembly systems, Operations Research, 40, 126-141, 1992.

2. D. Bertsimas, C. Teo and R. Vohra. Nonlinear relaxations and improved randomized approximation algorithms for multicut problems, Proc. 4th IPCO Conference, 29-39, 1995.

3. D. Bertsimas and R. Vohra. Linear programming relaxations, approximation algorithms and randomization : a unified view of covering problems, Preprint 1994.

4. G. Dobson. The Economic Lot-Scheduling Problem: Achieving Feasibility using Time-Varying Lot Sizes, Operations Research, 35, 764-771, 1987.

5. A. Federgruen, M. Queyranne and Y.S. Zheng. Simple power-of-two policies are close to optimal in a general class of production/distribution system with general joint setup costs, Mathematics of Operations Research, 17, 4, 1992. 
6. A. Federgruen and Y.S. Zheng. Optimal power-of-two replenishment strategies in capacitated general production/distribution networks, Management Science, 39, 6, 710-727, 1993.

7. M.X. Goemans and David Williamson. A new 3/4 approximation algorithm for MAX SAT, Proc. 3rd IPCO Conference, 313-321, 1993.

8. M.X. Goemans and David Williamson. .878 approximation algorithms for MAXCUT and MAX 2SAT, Proc. 26th Annual ACM STOC, 422-431, 1994.

9. D. Hochbaum and G. Shanthikumar. Convex separable optimization is not much harder than linear optimization, Journal of ACM, 37, 843-861, 1990.

10. P. Jackson, W. Maxwell and J. Muckstadt. The joint replenishment problem with power-of-two restriction. AIIE Trans., 17, 25-32, 1985.

11. P. Jackson and R. Roundy. Minimizing separable convex objective on arbitrarily directed trees of variable upperbound constraints, Mathematics of Operations Research, 16, 504-533, 1991.

12. Lu Lu and M. Posner. Approximation procedures for the one-warehouse multiretailer system. management Science, 40, 1305-1316, 1994.

13. W.L. Maxwell and J.A. Muckstadt. Establishing consistent and realistic reorder intervals in Production-distribution systems, Operations Research, 33, 1316-1341, 1985.

14. J.A. Muckstadt and R.O. Roundy. Analysis of Multisatage Production Systems, in: S.C. Graves, A.H.G. Rinnooy Kan and P.H. Zipkin (ed.) Logistics of Production and Inventory, North Holland, 59-131, 1993.

15. M. Queyranne. Finding 94\%-effective policies in linear time for some production/inventory systems, Unpublished manuscript, 1987.

16. P. Raghavan and C. Thompson. Randomized rounding : a technique for provably good algorithms and algorihmic proofs, Combinatorica 7, 365-374, 1987.

17. M. Rosenblatt and M. Kaspi. A dynamic programming algorithm for joint replenishment under general order cost functions, Management Science, 31, 369-373, 1985.

18. R.O. Roundy. 98\% Effective integer-ratio lot-sizing for one warehouse multiretailer systems, Management Science, 31(11),1416-1430, 1985.

19. R.O. Roundy. A $98 \%$ Effective lot-sizing rule for a multi-product, multi-stage production inventory system, Mathematics of Operations Research, 11, 699-727, 1986.

20. R.O. Roundy. Rounding off to powers of two in continuous relaxations of capacitated lot sizing problems, Management Science, 35, 1433-1442, 1989.

21. Y.S. Zheng. Replenishment strategies for production/distribution networks with general joint setup costs, Ph.D. Thesis, Columbia University, New York, 1987. 